\title{
Looking for Hypothetic Flora on Venus
}

\author{
Leonid Ksanfomality
}

Space research institute of the RAS. Profsoyuznaya str. 84/32, Moscow 117997, Russia

*Corresponding Author: ksanf@iki.rssi.ru

Copyright (C) 2013 Horizon Research Publishing All rights reserved.

\begin{abstract}
The planet Venus, with its dense and hot (735 $\mathrm{K})$ oxygen-free atmosphere of $\mathrm{CO}_{2}$ (mostly), having a high pressure of $9.2 \mathrm{MPa}$ at the surface can be a natural laboratory for studies of extraterrestrial life different from that of the Earth. On October 22/25, 1975 and March 1/5, 1982, experiments in television photography instrumented by the landers Venera-9, $-10,-13$ and -14 [1], yielded in large number of panoramas of the Venus surface (or their fragments) at the landing site. Over the past 31 and 38 years, no similar missions have been sent to Venus. Discovery and characterizations of extra solar planets suppose that some of them possess physical conditions close to those of Venus. In connection with the interest in what kind of life is possible existing on some of the exoplanets, the VENERA panoramas that fit for analysis were re-processed and re-examined. A few relatively large objects were found with size ranging from a decimeter to half meter, both terramorphic or with unusual morphology. The objects were observed in some images, but were absent in the other or altered their shape. Some of them were reviewed in Ksanfomality, 2012. One may suppose that the only source of energy for this hypothetic life is photosinthesis produced by plants. That's why important is a search of Venusian flora. The article presents some of the obtained results.
\end{abstract}

Keywords Venus, VENERA Missions, Extraterrestrial Life, Exoplanets

\section{Introduction}

The search for "habitable zones" in extrasolar planetary systems is based on the postulate on "normal" physical conditions, i.e., the pressure, temperature, and maybe atmospheric composition similar to those on Earth. But could not such an approach be considered as a "terrestrial chauvinism"? Observations of extrasolar planets show that among them there should be such bodies where the physical conditions are close to those on the planet Venus. Therefore, Venus itself, with its dense and hot $(735 \mathrm{~K})$ oxygen-free atmosphere of $\mathrm{CO}_{2}$, having a high pressure $(9.2 \mathrm{MPa})$, could be a natural laboratory for studies of this kind. The only existing data on the planet's surface are still the results obtained by the Venera landers in the 1970s and 1980s. The TV experiments on VENERA landers (October, 1975 and March, 1982) delivered 41 panoramas of Venus (or their fragments) [1-3]. There have not been any similar missions to Venus in the subsequent 38 and 31 years. In the absence of new landing missions to Venus, the results of these missions are unique. They have been studied anew, including the panoramas not analyzed earlier.

A few relatively large objects, from a decimeter to half a meter in size, with an unusual morphology were found, which slowly moved or slightly changed their shape. A number of Venusian fauna candidates reach about ten for to-day. Their emergence by chance could hardly be explained by noise. Part of these objects were observed in some images but were absent in others.

The present paper is devoted mainly to the hypothetical flora of Venus. Entities that can be attributed to hypothetical fauna of the planet were discussed in [2-4] and other papers. (Strange enough, a part of hypothetical fauna is terramorphic). A number of Venusian fauna found candidates reaches about ten for to-day. In contrast to the Earth's biosphere, where the heterotrophic fauna relies on the existence of an abundant flora and the oxidant that is reach in atmosphere, mechanisms of energy supply for hypothetical fauna of Venus are unknown. Oxygen concentration in the atmosphere is very low (about $10 \mathrm{ppm}$ ). One may suppose the only source of energy is photosinthesis produced by plants. Based on analogy with the Earth's biosphere, it has been suggested that the hypothetical fauna of Venus exists due to its flora. Efforts have been made looking for it. Signs of it, seems, have been found indeed. How the Venusian biophysics is organized is unknown and it is too early proposing any hypothesis about.

This paper presents some of the results obtained and analyzes indications testifying to real detection of these objects. Few examples are shown below.

\section{Information on the Experiment}

The results presented below correspond to the missions from which images were obtained (VENERA 9, 10, 13, 14), among which the lander VENERA-13 worked for a much longer time than the others (127 minutes). The coordinates of 
its landing point were $7.5^{\circ} \mathrm{S}$ and $303.5^{\circ} \mathrm{E}$, and the altitude of this point over the level of the radius $6051 \mathrm{~km}$ was $1.9 \mathrm{~km}$ [2]. The temperature was $735 \mathrm{~K}\left(462^{\circ} \mathrm{C}\right)$, the pressure $8.87 \mathrm{MPa}$, the corresponding density of the atmosphere was $59.5 \mathrm{~kg} / \mathrm{m}^{3}$, and it consists of $\mathrm{CO}_{2}(96.5 \%)$ and $\mathrm{N}_{2}(3.5 \%)$. The local time was $10 \mathrm{o}$ 'clock in the morning with a solar zenith distance of $37^{\circ}$. The illumination (by scattered solar light) amounted to 3-3.5 klx [2]. Two mechano-optic cameras were mounted on opposite sides of the lander [1]. VENERA-14 landed $800 \mathrm{~km}$ eastward from it. Each lander VENERA -13 and VENERA-14 was equipped by two TV-cameras. The camera's aperture was located at a height of $0.9 \mathrm{~m}$ over the surface, and the upper boundary of the panoramas at the center was at a distance of $2 \mathrm{~m}$ from the spacecraft center. The axes of the scanning cameras were placed at an angle of $50^{\circ}$ from the vertical line, which allowed for resolving millimeter details of the surface in the immediate vicinity of the lander and those of about $10 \mathrm{~m}$ near the mathematical horizon (at a distance of $3.3 \mathrm{~km}$ on a level surface). However, the tilt of the camera axis led to geometric distortions. If one transforms the image in such a way as to correct the line of horizon, the panorama becomes a figure bounded by two arcs (Fig.1).

Venera 13 gave three full black-and-white (BW) and color-divided panoramas ( $\mathrm{R}$ red, G green, and B blue), covering an angle of $180^{\circ}$. Three series of images have been consecutively obtained, numbered 1,2 , and 6 . The images from Venera-13 presented in the text are designated as, for instance, G6 (the third series, green). The blue images are useless because the planetary atmosphere is opaque to blue light.

The spectral intervals (Fig.2) were 410 to $750 \mathrm{~nm}$ (no filter), 490 to $610 \mathrm{~nm}$ (a green filter), and 590 to $720 \mathrm{~nm}$ (a red filter). Obtaining a single panorama (in the TIFF format) with its simultaneous transfer through a satellite of Venus took 13 minutes. A table with time locations of the images was presented in [3]. Each image consisted of 1000 vertical lines (11 arc minutes each), with a resolution of 252 pixels per line, 211 pixels on the active part (252 pixels including housekeeping). With the scanning duration of $0.78 \mathrm{~s}$ per line, each pixel was transmitted for $3.3 \mathrm{~ms}$. During the 38 and 31 years that have elapsed since the Venera missions were completed, the author repeatedly returned to the images obtained in order to reveal any unusual elements. The images published immediately after the experiments were created by combining black-and-white and colored panoramas. Apart from these, there are other primary images in which the author tried to reveal any distinctions in consecutive panoramas (emergence or disappearance of details or changes in their view) and to come to an understanding of the causes of such changes (e.g., the wind). Another indication of objects to be sought is connected with their morphology, distinguishing their shape from that of ordinary details of the surface. The suggested work is devoted to some results of studying the images transmitted by the landers Venera-9, Venera-13 and Venera-14.

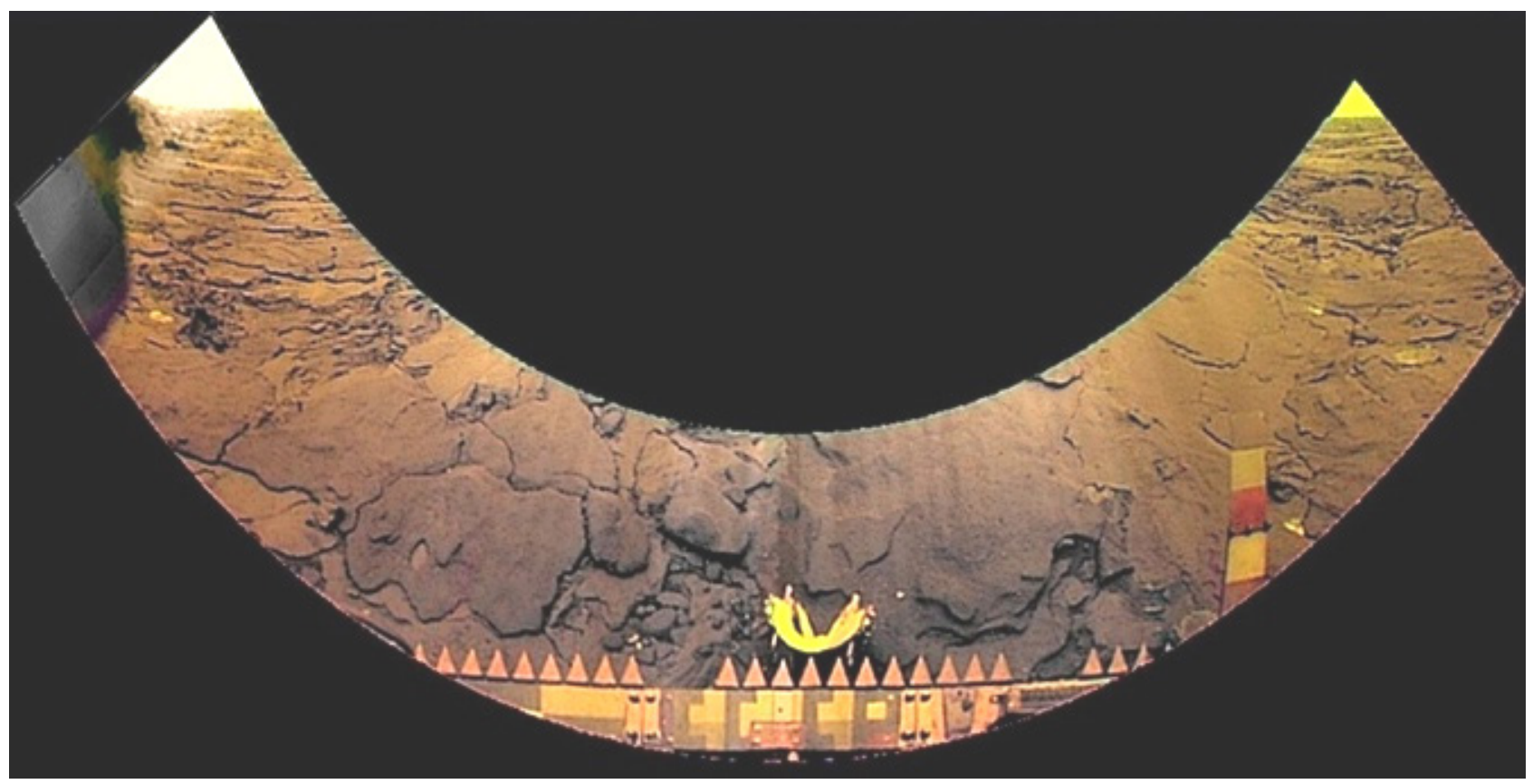

Figure 1. Re-processed VENERA-14 panorama with corrected geometry (2012). 



Figure 2. Spectral sensitivity of the landers cameras; VENERA-9 (A) and VENERA-13, -14 (B)

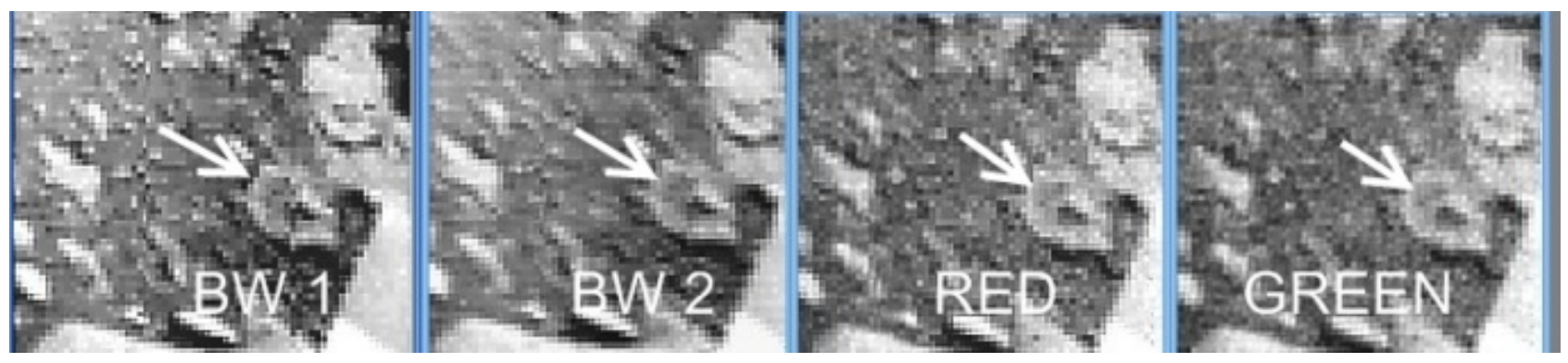

Figure 3. Fragments of the VENERA-13 panoramas with the "wood fungus" object, without processing.

\section{Looking for Signs of Hypothetic Flora on the Venera Panoramas}

The present paper continues articles published previously and deals with analysis of Venusian surface images. In the 41 images registered by all VENERA landers, there are 10 or 11 unusual objects. Descriptions of part of them were considered in papers [2-4] or are being analyzed and prepared for publication. It is necessary to stress that in processing the original images, any kinds of retouching additional drawing, or correction were completely excluded. Any application of nonlinear software tools was excluded as well. The contrast and brightness of the images were corrected. If it was allowed by the image structure, the operation of "blurring" was applied, together with "sharpening" of the standard Microsoft Office software of the Windows system. An analysis of details of the surface images made it possible to single out a few objects satisfying the above criteria. For convenience of presentation, they are given conditional names which are absolutely conditional. The most of the paper deals with flora-like findings on the VENERA panoramas.

\subsection{Hypothetic Mushroom-like Objects}

As a result of the panoramas newly processed the quality of the images was noticeably improved. Two of the most interesting findings on the panorama obtained with camera 1 of the VENERA-13 lander seem relate to hypothetic Venusian flora. These objects were investigated first in 2012-2013. With the improved methods of image processing, contours of previously unclear details became sharper. In this section, novel objects "wood fungus" and "a mushroom" belonging to hypothetical living forms are presented. Prior to the new image processing, these objects have not attracted any attention, although one of them has been located in the very foreground of the VENERA-13 panorama.

Four fragments of non-processed versions of panoramas of the landing site of the VENERA-13 are shown in Fig.3. Two black-and-white BW 1 and BW 2, Red and Green images were obtained during the first $87 \mathrm{~min}$ of the mission. A novel object is seen in the fragments of sequential panoramas, close to the color test panel. May be another similar feature is placed above it in the image. When processed by the method of correlative stacking [5] it results in an image shown in Fig.4. The feature (2) has a regular shape with bright spots placed along its edges. Its shape resembles something like a terrestrial wood fungus. So it has been called "wood fungus". The length of the feature is about 6-7 cm. There are no stones of this shape in vicinity.

The second novel object whose shape resembles a mushroom is located in the foreground at a distance of 15 to $20 \mathrm{~cm}$ from the buffer of the lander (see combined Fig.5). The object is elevated above the surface by about $3 \mathrm{~cm}$; 
however, its support is not visible.

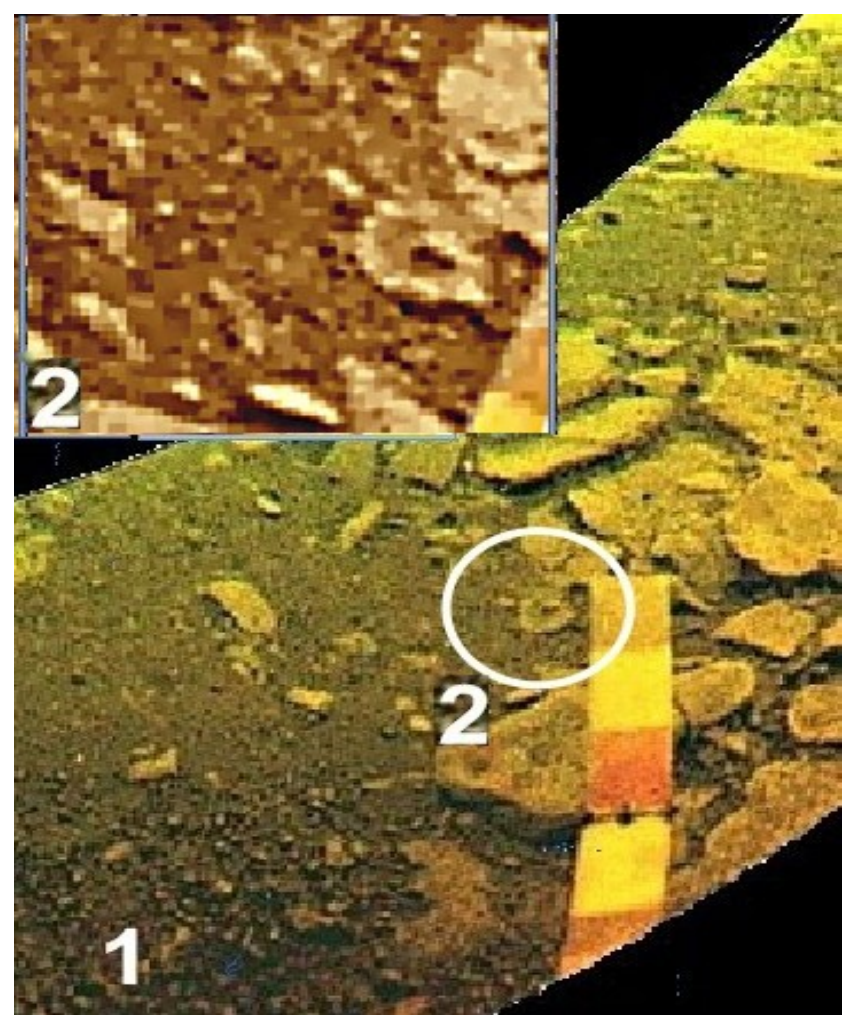

Figure 4. Position of the "wood fungus" object (2) on the fragment (1) of the VENERA-13 panorama.

The diameter of the "mushroom" attains, approximately, 8 $\mathrm{cm}$. It is clearly seen on all sequential panoramas of VENERA-13. In Fig. 5, 1, the mushroom is the brightest object in the central part of the panorama. Comparing the brightness of this object with the lighter periphery of the figure, one should keep in mind that the object resides in the shadow region made by the parachute panel of the landing module. The color image (Fig. 5, 1) is composed of black-and-white and color-divided red and green primary panoramas. Six sequential images were processed by the method of correlative stacking again, including all available panoramas. Three of the resulting versions of black-and-white images are shown in Fig. 5, 2. In each case, the radial tent-shaped folded structure of the object is seen. The size of the mushroom is commensurable with small "hesperos" found earlier [4]. For the 1.5-hour observation of the Venusian surface, no attributes that could testify to motion of the object were found. One may suspect a small motion of the "wood fungus", about a centimeter, seen using a blink-comparator. However the resolution is too poor for a confident conclusion.

Apparently, one may relate these objects to Venusian flora (not discussing a difference between the flora and mushrooms.) By virtue of the fortunate close position of the "mushroom", its structural details are clearly distinguishable. The "mushroom" object, apparently, relates to Venusian flora indeed. This allows associate its properties with the most noticeable manifestations of terramorphism, which again indicates certain unknown yet biological regularities [4]. By virtue of the small size of the mushroom, it is hard to observe other similar objects remote from the camera at a greater distance. Two small black points at the upper part of the "mushroom" are seen repeatedly in all images.

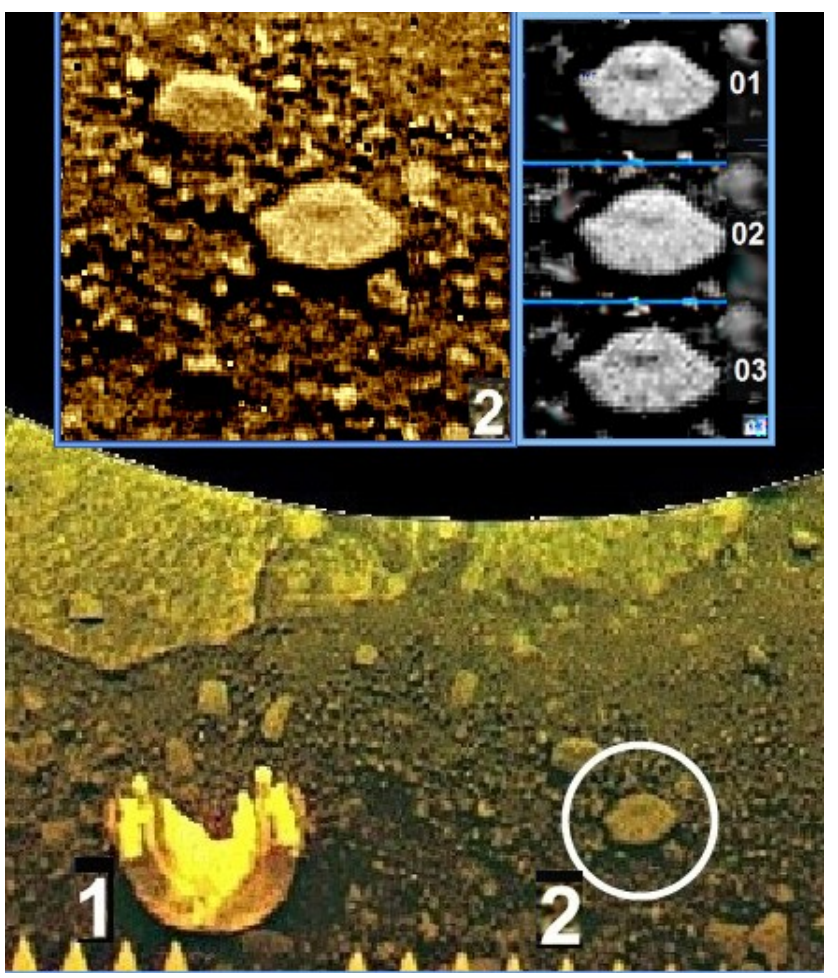

Figure 5. Position of the tent-shaped object "mushroom" (2) on the fragment 1 of the VENERA-13 panorama. Three black-and-white figures demonstrate a radially folded structure of the object resembling Earth' mushrooms. The white feature is the camera's lid.

\subsection{The "Anemos"}

On the panoramic pictures of VENERA-9 and VENERA-13, -14 a few other probable samples of the planet's flora have been found. The conditions under which series 1, 2, and 6 of the VENERA-13 panoramas were obtained have been considered in detail in [2,3]. In all cases the objects under consideration were separated from the camera lenses by distances close to $2 \mathrm{~m}$. A pair of objects that attracted attention by changing their positions on consecutive images is marked by an arrow on the fragment of the first BW panorama of VENERA-13 (Fig. 6). The objects have received the name "anemos" because of their similarity to the tentacles of anemones, sea polyps living on stones in shallow waters. Two anemos, about 5-7 cm long, are stretched from the stone in the $7 \mathrm{o}$ 'clock direction and do not differ from the other elements of the picture except that the surface around them appears somewhat foggy. However, in the subsequent pictures (Fig. 7), it can be seen that over 1.5 hours the "anemos" move while the position of their upper point (at the stone) apparently does not change. The time at which the pictures were obtained (the beginning of scanning each panorama) is indicated below the pictures. 


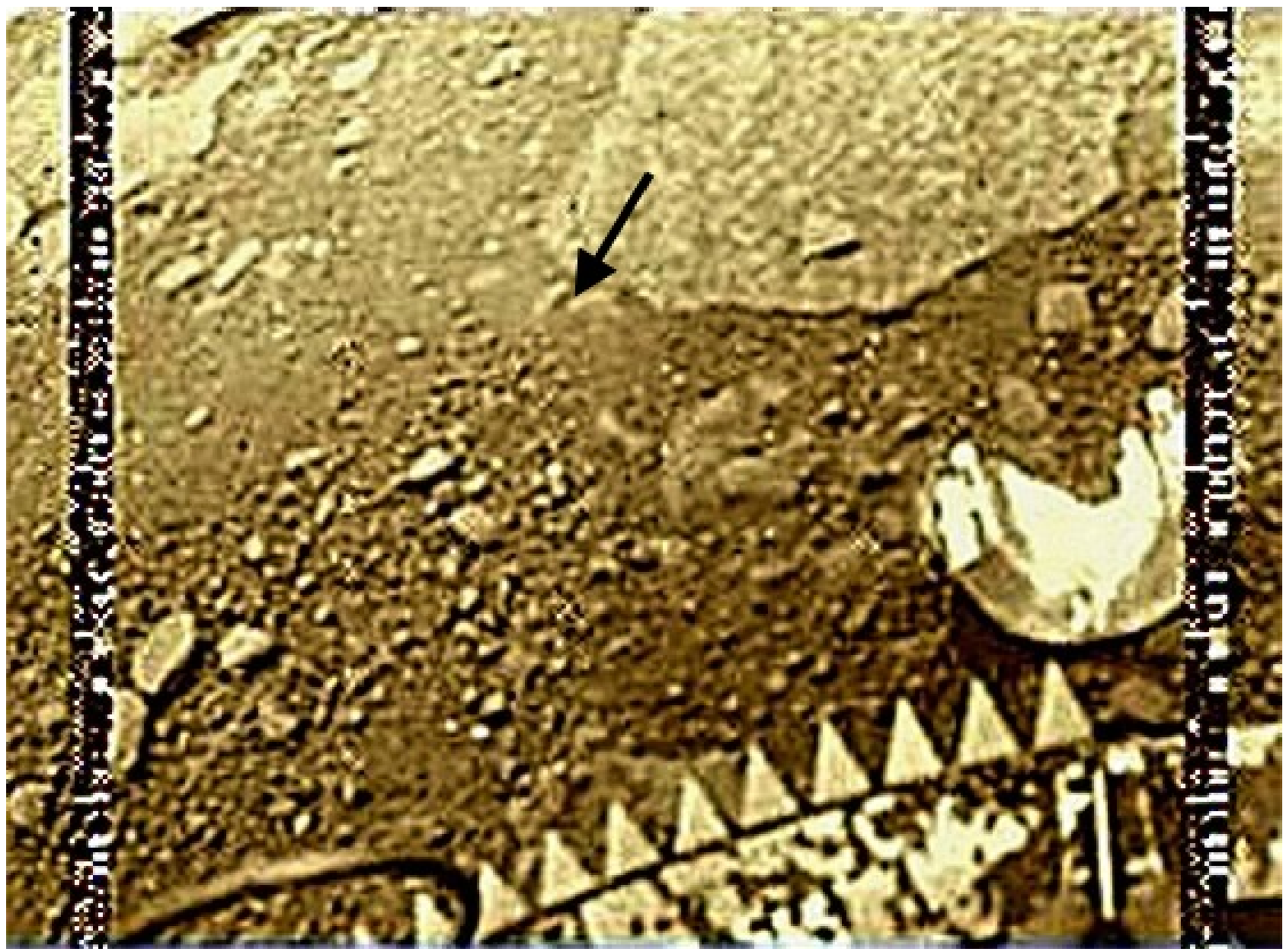

Figure 6. A fragment of the first panorama of VENERA-13. The "anemos" objects are shown by the arrow. The pair of objects changes their positions in the subsequent panoramas of VENERA-13.

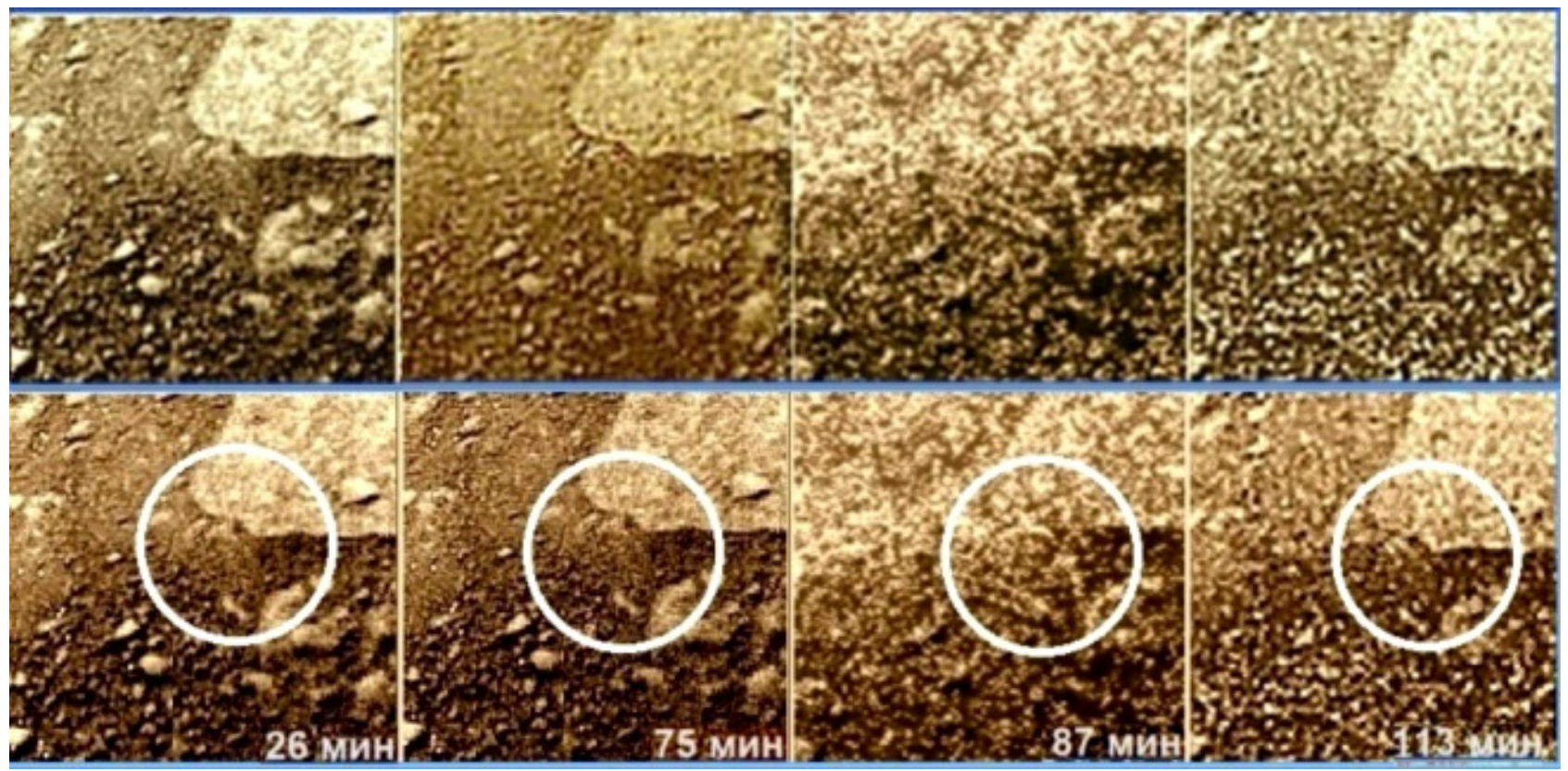

Figure 7. The pair of "anemos" objects which abruptly change their positions in the consecutively obtained panoramas of VENERA-13 over $1.5 \mathrm{~h}$. On the lower panel the "anemones" are marked by circles. It is shown the time of obtaining of the "anemos" image in each of the four pictures. 
The contrast of the pictures has been enhanced. The "anemos" position is designated by a white circle. Since the camera's angular resolution was equal to $11^{\prime}$, the size of each single pixel corresponding to a distance of $2 \mathrm{~m}$ was equal to $6.4 \mathrm{~mm}$; therefore, the precision of the images is restricted.

Along with the panorama fragments, Fig. 7 in lower panel shows the changes of the "anemos" position marked by circles. In Fig. 7, the frame of 26 min, the "anemos" " position is the same as in Fig. 6. For about $1 \mathrm{~h}$, until the frame at 75 min, the anemos slightly stretched and bended down, to the direction of 6 o'clock. Then, 13 min later, in the 3rd frame (87 $\mathrm{min})$, the "anemos" were stretched substantially and rose above the surface, forming a half-ring about $10 \mathrm{~cm}$ in diameter.

The thickness of the stretched "anemo" was not larger than 3 to 5 pixels, i.e., 1.5 to $2 \mathrm{~cm}$. The panorama V-13-1-6 BW (87 min) is noisier than the previous and subsequent ones, and the left side "anemo" (behind the first one) is seen poorly. Here the direction of the "anemos" is approximately the same as in the first frame.

The left end of the "anemo," for some reason, rests against the "scorpion" [2], which has appeared just in this picture (87 $\mathrm{min})$. Further, $26 \mathrm{~min}$ later, in the 4th frame (113 min), both "anemos" together turned by almost 90 degrees in the direction of 5 o'clock. Their lengths remained almost the same as in the frame of $87 \mathrm{~min}$. The nature of the "anemos" certainly remains unknown. Since the upper part of the objects in Figs. 6 and 7 remains at rest, one can assume that they belong to the hypothetic flora. But the above restricted data are of course insufficient for a confident conclusion.

\subsubsection{Are "Anemos" and the "Scorpion" Associated?}

The "scorpion" does not belong to Venusian flora. However, there is no explanation of the "anemos" behavior at the moment of the "scorpion" appearance and their possible association. It should be reminded that the "scorpion" was found on the panorama BW 6, considered in [2]. Its scanning began at the 87th minute of operation. From the results of the special study it was concluded that little white dots in the BW 6 image, with a brightness level close to saturation, correspond to a noise of electromagnetic origin that emerged either in the overheated electronics or on the lander-to-orbiter line, which could be explained as a short-term (within a few milliseconds) loss of communication. There was no such noise in eight previous panoramas, but now the electronics was already dangerously heated. The images were transmitted in a negative format; therefore the result of an incidental loss of the signal was a fault in the form of a white dot. So, the dots are mainly indeed results of electric noise. The noise density is not very high and the appearance of details of the surface is easily restored by the image processing.

The object, conditionally called "the scorpion," was discovered at early stage of the study. A fragment of the panorama with "the scorpion" is shown in Fig.8. Before the BW 6 panorama the instrument had already been working for 1 hour and 27 minutes (moment of the start of scanning the BW 6 image); therefore, the first suggestion was that this regular structure is a product of destruction of some part of the lander itself. But the VENERA-13 lander continued to work for almost a whole hour after that, indicating that there could not be any destruction, otherwise all instruments would have failed due to catastrophic overheating. An analysis of the technical documents showed that all external operations (such as throwing the camera's lids away, operation of the drilling setup) terminated in less than 30 minutes. The assumption of a split detail is also in conflict with the fact that the object is absent in the subsequent images.

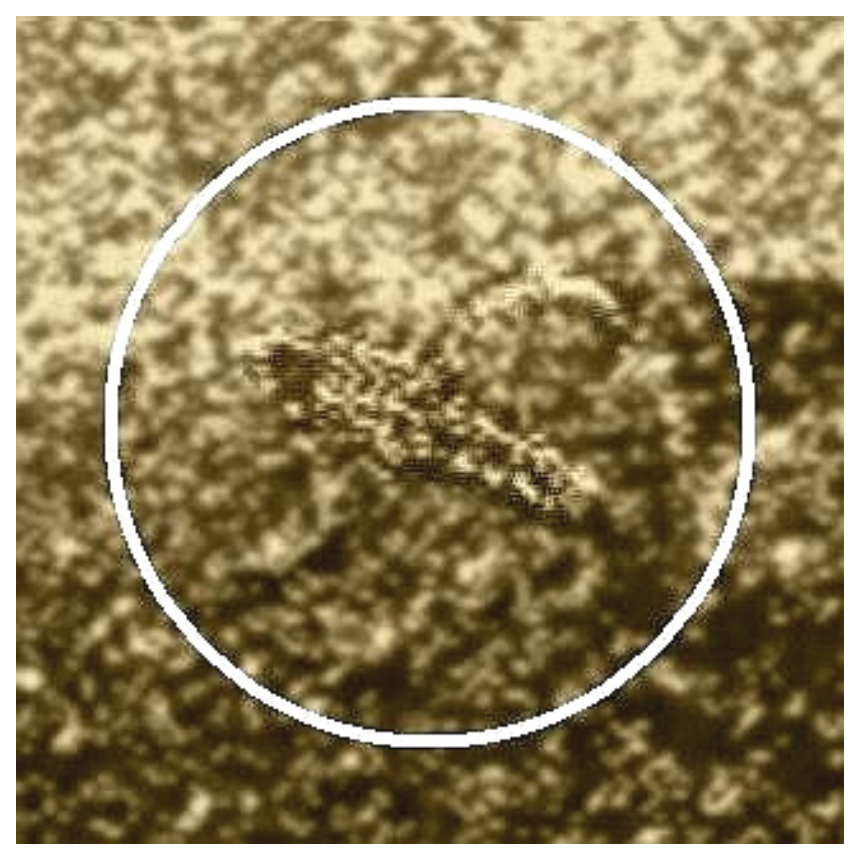

Figure 8. The object "scorpion" is $15-17 \mathrm{~cm}$ long.

The history of the emergence and disappearance of the "scorpion" is illustrated in Fig. 9. In images obtained before the 87th minute, the object is absent. The object is absent as well in the later image G6 (113 to $126 \mathrm{~min}$ ). A likely cause may be in that, if the object moved, then, as it went away to a distance of 3 to 4 meters, it became indistinguishable from stones. At such a distance, as a minimum, the object should have moved away in 26 minutes, the time up to the next panorama G6 in Fig. 9. In the course of scanning, the image of a moving object can be distorted. The image fragment with the "scorpion" was obtained in a period of 32 seconds.

The object motion could cause, for instance, its seeming lengthening or shortening in the image.

The "scorpion" has a length of 15 to $17 \mathrm{~cm}$ and a complicated structure resembling some terrestrial Arachnida or insects. In its immediate vicinity in the panorama $6 \mathrm{BW}$, there emerged an "anemo" like a half-ring, of the same size. 


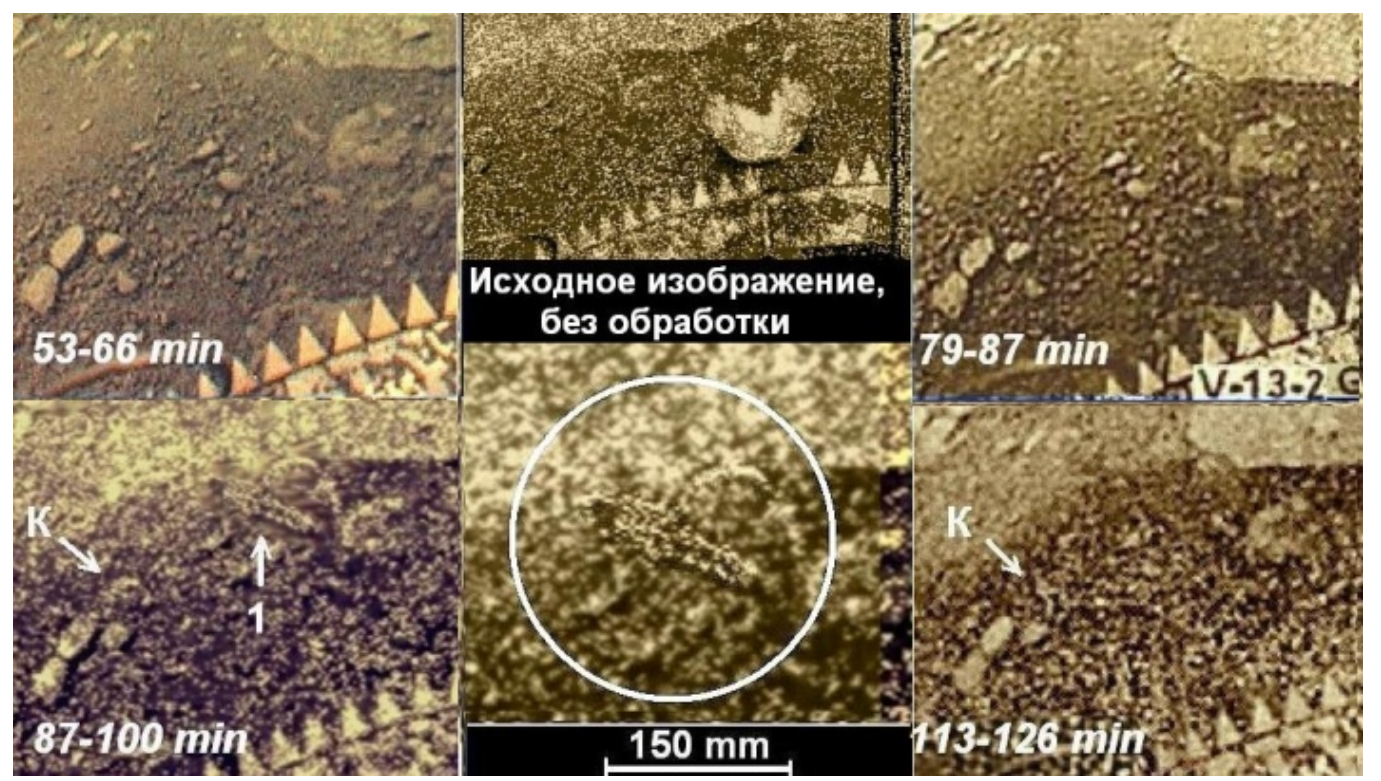

Figure 9. The "scorpion" appeared in the panorama BW 6 obtained from the 87th to the 100th minutes. In images obtained before the 87th and after the 113 th $\mathrm{min}$, the object is absent. In the images of minutes $87-100$ and 113-126 min, to the left, in the group of stones, there emerged a new object K with a changing shape. The central part of the figure, at the bottom, shows the result of the "scorpion" image processing and its size.

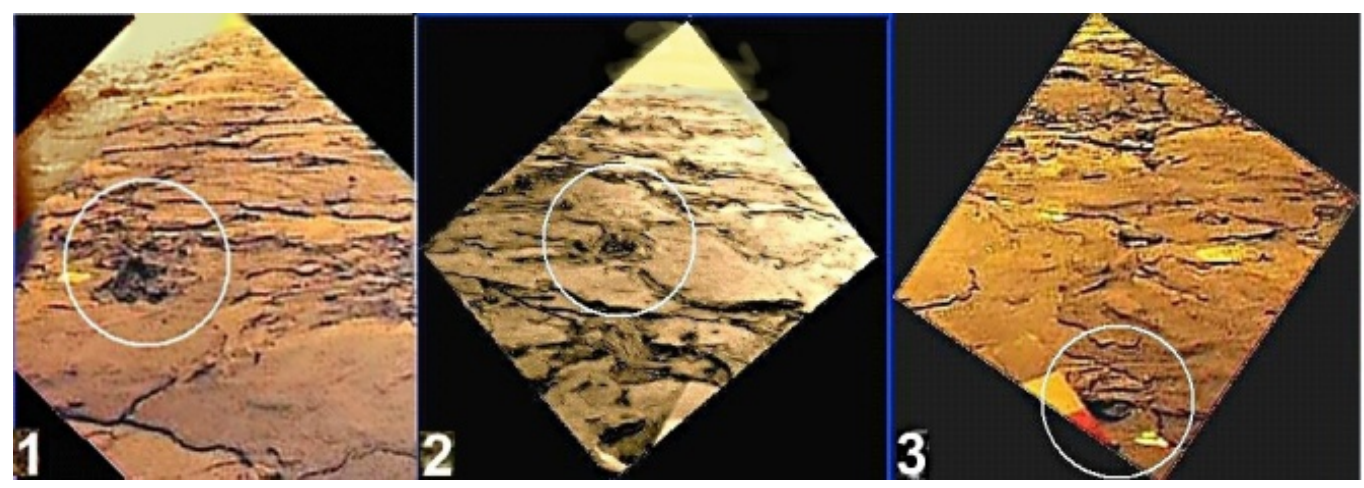

Figure 10. Objects "piles" of unknown nature at the landing site of VENERA-14 may resemble rare bushes on dried-up terrestrial salines.

The complex and regular shape of the "scorpion" cannot be a result of a random combination of light, half-tone, and dark points. The image of the "scorpion" consists of 940 points, while the number of points in the whole panorama, covering $177^{\circ}$, is $2.08 \cdot 10^{5}$. The probability $p$ of forming such an image, if we count only the number of combinations, is vanishingly small, $p<10^{-100}$, and is in fact excluded. In addition, there is a physical indication of "scorpion's" reality: an analysis reveals a shadow under the object. Shadows certainly cannot form near a random combination of points. A shadow shows that the object has a relief and is located over the surface.

Most probably, the emergence and then disappearance of the "scorpion" are connected with destruction and lateral ejection of soil in the course of landing rather than a direct influence of the wind [2]. The vertical speed of the spacecraft at landing, found by a dynamical method was $7-8 \mathrm{~m} / \mathrm{s}$, while the lateral speed was approximately the same as that of the wind $(0.3$ to $0.5 \mathrm{~m} / \mathrm{s})$. The stroke amounted to $50 \mathrm{~g}$ of Venus. The lander destroyed the soil to a depth of about $5 \mathrm{~cm}$, threw it aside, and the soil could have covered the "scorpion." The place where the "scorpion" appeared was studied in the whole sequence of panoramas, from the $7^{\text {th }}$ to the $119^{\text {th }}$ minute. At first, a shallow gutter of about $100 \mathrm{~mm}$ long is seen on the soil thrown out. Then the sides of the gutter are lifted, and its length grows to about $150 \mathrm{~mm}$. The gutter orientation is the same as that of the "scorpion". In an hour, the regular structure of the "scorpion" emerged from the gutter. At the 93rd minute, the "scorpion" probably completely got out of the soil that had covered it, whose whole layer was not thicker than 1 to $2 \mathrm{~cm}$. At the 119th minute, it had already gone. Thus, the object needed about an hour and a half for the "rescue" operation. This apparently points to its restricted physical abilities.

The pair of "anemos" semi-rings seems are in action with the "scorpion", as seen in Figs.8-9. They touch the "scorpion's" body. There is no evidence, is it just a coincidence of the stage of elongation of "anemos" with the "scorpion" appearance, or not.

\section{3. "PILES"}

Some interesting but incomprehensible details have been 
found in the panoramas of VENERA-14, on both sides of the spacecraft. Figure 10 shows three such objects, which certainly may be simply some geological formations.

Their unusual nature is that they resemble rare bushes on dried-up terrestrial saline lands. On a comparatively uniform and solid surface, at a distance of about $2 \mathrm{~m}$ from the spacecraft, there is a low object endowed with relief, less than half a meter in size (Fig.10, frame 1). Its contour slightly resembles a lichen (if one forgets the incomparable size), while central part has the form of a small hill or pile. But it is also possible to say that the object resembles a dried-up pile of mud thrown from somewhere, for example, a semi-liquid volcanic emission. The existence of volcanos on Venus has been established, but they are not so numerous that one could suppose the occasional existence of a nearby volcano, particularly because the surface seems to be very old. The object color is darker than that of the ambient surface. May be, something of this kind has also been detected by the TV camera on the opposite side of the VENERA-14 lander (frame 2), but the object is more distant, and it is difficult to make out the details. Quite close to the landing buffer, there is one more, smaller round object lying on the surface (frame 3 ), of about $15 \mathrm{~cm}$ in size. It reminds a nut. As far as it can be judged, it has a shape different from that of the objects shown in frames 1 and 2, nor does it look like one of the geological formations on the ambient surface. Despite its regular shape it is not excluded that "piles" may be a stony fragment that broke due to the shock while landing (at a speed of $7 \mathrm{~m} / \mathrm{s}$ ). It should be noted that listed formations have been found at the landing site of VENERA-14 only. It can hardly be connected with the local altitude: the VENERA-14 zone is at an altitude of $1.3 \mathrm{~km}$, while that of VENERA-13 is at an altitude of 1.9 $\mathrm{km}$ over the level of the mean planetary radius.

\section{The "Black Rag"}

Along side of camera 2 of the VENERA-13 lander, there was an instrument for measuring the mechanical properties of the ground [6]. After landing, a catch was released, a spring made a pendulum-type truss straighten up, and a measuring cone (hammer) drove into the ground (Fig.11). The truss length was equal to $600 \mathrm{~mm}$. Since the mission objectives included an analysis of small components of the atmosphere and the soil, the presence of any organic or carbonizing materials on the instrument and on the outer parts of the spacecraft itself was excluded, as was separation of any films from the lander.

These conditions were paid great attention in a factory test. After landing, the covers of TV cameras (white half-cylinders in the figures) were thrown away by means of pyrocartridges, and several other instruments were released.

In the first image of Fig.11 (obtained in the interval of 0 to 13 minutes from the beginning of the operation), it can be seen that around the cone, along its whole height, there emerged a vertically stretched object of unknown origin, wrapping the cone up, a "black rag", with a size of 60 to 80 $\mathrm{mm}$. In the subsequent images, obtained at 27 minutes and later, this object had completely disappeared. From comparison with other images, one can assume that the emergence of the black object is somehow connected with ground destruction by the measuring cone. The object cannot be a fault of the panorama: in Fig. 11 it is apparent that details of the mechanism are projected on the object while other details are visible through the "rag". Looking for possible explanation of the appearance of the "black rag", a suggestion was proposed that when the soil crust was crashed by the cone, some possible gaseous stuff appeared from the soil and condensed on the cone. However, why it condensed on the cone only?

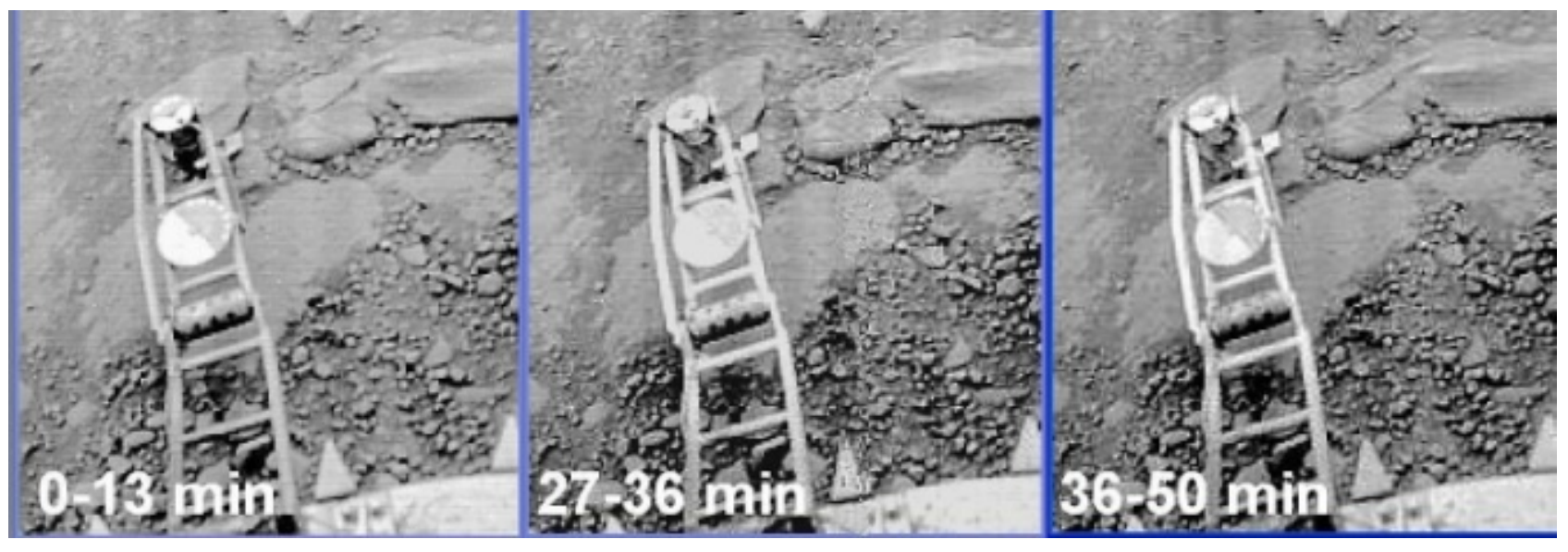

Figure 11. The unknown object "black rag" emerged in the first 13 minutes after landing around the conical measuring hammer which partly penetrated into the soil. The subsequent images (obtained from the 27th to the 50th minute of operation) show a clean surface of the conical hammer, while the object "black rag" or its fragments are absent. 


\section{A "Stalk"}

Flora of Venus certainly applies to "stalk" (VENERA-14 panorama). As for the VENERA-13, VENERA-14 panorama contains several objects of unusual shape. In all panoramas, both black-and-white and color, when considered carefully, in the central part of the fragment a dark slanted line or narrow band is seen, corresponding to the unknown object. The band is not related to parts or technical characteristics of the lander, as well as to interferences in radio communications. The line did not change its position relative to the lander and to the surface micro-relief, during the whole duration of its work, almost 2 hours. The treated fragment is shown at the left part of Fig. 12 , in the center. The entity is situated close to the landing buffer, approximately $15 \mathrm{~cm}$ from it, making it possible to highlight details. The facility has a vertical knotty structure that reminiscent extremely the structure of the stems of terrestrial plants. It ends with a round tip of the bulge, a "bud", with a bright dot in the center. The lower part of the "stem" is based on a group of 4 elements reminiscent of quatrefoil. The same piece of the VENERA-14 panorama, but with the background blurred is presented at the right part of Figure 12. Two "leaves" show their radial structure. Of course, he "quatrefoil" can be jaust a random position of debris. But the "stem" is protruding from the very center of the "quatrefoil". Set the color of the items is not possible, because the fragment of panorama is black and white. Height of the "stem" is determined by the cosin of angle at which the camera registers it, about $75-80^{\circ}$. Therefore, its height is $55-60 \mathrm{~cm}$. In Fig. 12 it is seen from the above. The "stem", along with "mushroom" are typical terramorphic objects.

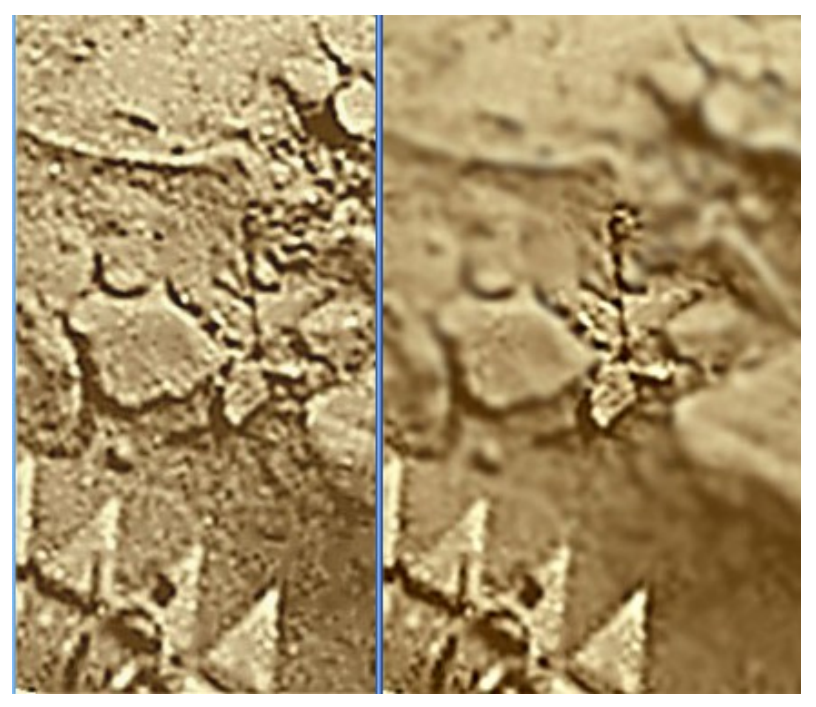

Figure 12. A "stalk" found in the VENERA-14 panorama

\section{Discussion}

The hypothesis on possible life (in unknown forms) on the surface of the planet Venus [2-4] at high temperatures (735
$\mathrm{K})$ is quite a new subject. Numerous research articles (e.g., [7]) have repeatedly discussed the possible existence of life under the conditions of Venus's $\mathrm{CO}_{2}$ atmosphere without oxygen, but only high in the atmosphere, at a level whose temperatures roughly correspond to those on Earth. The authors arrived at the conclusion that the possibility of life on Venus is not excluded, for instance, in the form of microbiological objects floating in the atmosphere. Quite another thing is possible life at high temperatures like those near the surface of Venus. It would require an unknown metabolism of quite a different type than the one used by terrestrial organisms. The possible existence of such unknown forms was pointed out by Jones [8] and earlier by the present author [9]. Without deeply developing this theme, Jones writes: "It does not make sense to rely in our approach too literally on the life pattern that exists on Earth, i.e., that with RNA, DNA, and a particular set of proteins; otherwise, we are at risk of missing other opportunities. Even more than that, by following some research strategies we could perhaps discover life on the basis of quite a different chemical composition (e.g., without carbon and/or water)." Besides initially waterless life, one could also consider the assumption of life of the terrestrial type, which had emerged at early stages of the planet's history and adapted to very slowly changing conditions of gradually increasing temperature and pressure, developing on the planet over hundreds of millions of years $[2,4]$. The climate changed along with the loss of water in the process of a greenhouse run-away effect. The cosmogonical activity of such processes is indirectly indicated by the great abundance of deuterium in the traces of water on Venus, which is larger than the terrestrial $\mathrm{D} / \mathrm{H}$ ratio by a factor of 100 to 150 [10]. This abundance has emerged due to the weak selectivity of hydrogen losses as compared to deuterium, which has resulted in the observed abundance due to the immense scale of the process. The temperature range of 725 to $755 \mathrm{~K}$ near the planetary surface (depending on the relief) is certainly absolutely incompatible with the terrestrial forms of life, but, in essence, from a thermodynamic point of view, these conditions are not at all worse than those on Earth. Indeed, the chemical reactions and the corresponding chemical substances are unknown, but nobody has studied them. Chemical reactions are very active at high temperatures, while the source materials are almost the same on Earth and on Venus. In biophysics, many anaerobic organisms are well known. In many prokaryotes, photosynthesis occurs in a reaction where hydrogen sulfide $\mathrm{H}_{2} \mathrm{~S}$ instead of water is a donor of electrons. Many autotrophic prokaryotes, including the underground ones, use chemio synthesis instead of photo synthesis, producing the simplest carbohydrates which can be schematically designated as $\left(\mathrm{CH}_{2} \mathrm{O}\right)$. The principle of such a reaction appears as follows: $2 \mathrm{H}_{2} \mathrm{~S}+\mathrm{CO}_{2}+(\mathrm{C}) \Rightarrow 2\left(\mathrm{CH}_{2} \mathrm{O}\right)$ $+2 \mathrm{~S}$. Such a reaction does not release oxygen, while sulfur is of interest in that it is this chemical element that is a meteorologically active agent on Venus. There is no visible physical prohibition of life at high temperatures. Certainly, at high temperatures and in an environment without oxidation, 
any photosynthesis should probably be built on quite different, unknown biophysical mechanisms equivalent to the terrestrial one, although direct sunbeams, as a rule, do not reach the planetary surface. An interesting feature of the Venusian flora (as well as fauna) should be their adaptation to the very long duration of day and night [10]. The annual period of Venus (224.7 terrestrial days), combined with the rotation period (243 days), taking into account the inverse rotation, results in a duration of a sunny day of $T_{\text {sol }}=\left(T_{\text {sid }}{ }^{-1}+\right.$ $\left.T_{o r b}{ }^{-1}\right)^{-1}=116.8$ days. Since the rotation axis is almost normal to the orbital plane, day and night are equal to each other and last 58.4 days each. Seasonal effects are absent. Independently of a specific biophysical mechanism active on the surface of Venus, at temperatures of the falling $\left(T_{1}\right)$ and leaving $\left(T_{2}\right)$ radiation, the thermodynamic efficiency of the process should be slightly less than on Earth because $T_{2}=$ $290 \mathrm{~K}$ for the Earth and $T_{2}=735 \mathrm{~K}$ for Venus. Due to strong absorption of the blue-violet part of the spectrum in the atmosphere, the maximum of solar radiation is shifted on Venus to the green-orange domain, and, according to Wien's law, it corresponds to a lower effective temperature, $T_{1}=$ $4900 \mathrm{~K}$ (on Earth $T_{1}=5770 \mathrm{~K}$ ). It should be noted that thermodynamically, among the planets of the Earth's group, Mars possesses the most favorable conditions for life. The previous publications [2-4] give some ideas on Venus's hypothetic fauna. But objects of the planet's hypothetic flora are not numerous, and, moreover, some of them are hard to definitely classify the objects under question precisely as those of flora.

The Earth's heterotrophic fauna exist due to the existance of an abundant flora and the oxidant in atmosphere. However the oxygen concentration in the Venus atmosphere is very low (about $10 \mathrm{ppm}$ ). Same about the water vapor. Based on analogy with the Earth's biosphere, one may suppose the only source of energy of Venusian fauna are plants, which exsistance in turn is based on the photosinthesis. There is enough diffused sun light at the surface, up to $10 \mathrm{klk}$. It has been suggested that the hypothetical flora of Venus could be found in VENERA panoramas. Efforts have been made looking for it. Signs of hypothetical flora of Venus, seem, have been found indeed.

\section{Conclusions}

In TV experiments conducted on the surface of the planet Venus in 1975 and 1982 on the basis of the Soviet landers of the VENERA series, several tens of complete panoramas (or panorama fragments) of the Venusian surface were obtained. In the course of continued image processing based on modern methods the processing quality and the resolution of image details were improved noticeably. The papers [2-4] give certain ideas of Venus's hypothetical fauna. The existence of large objects of Venusian fauna discussed in [2-4] should be supported by some energy sources, and by analogy with the Earth's biosphere, it is possible to ascribe such sources to hypothetical autotrophic flora of Venus.
Besides the hypothesis that life could emerge in the circumstances of an initially waterless planet, one must also consider the assumption that life of the terrestrial type could emerge at early stages of the planet's history and gradually adapt to very slowly changing conditions of growing temperature and pressure, which developed over a few hundred million years. But objects of the planet's hypothetical flora in hands are not numerous and are not easily found. Moreover, it is hard to classify some objects under suspicion as precisely those of flora. As such representatives of the hypothetical flora, the objects named the "mushroom", "wood fungus", and the "anemos" are discussed, which are morphologically, but in different ways, similar to the Earth's living forms? An object of hypothetical Venusian flora, a "mushroom" was located relatively close to the camera lens. The object exhibits explicit similarity to terrestrial mushrooms and is supplied with folded cap. The "mushroom" has clearly expressed terramorphic attributes, which, probably, indicates biological regularities yet unknown at present [4]. Apart from them, on the landing spot of VENERA-14, some objects of unknown nature have been found, which were not detected at the landing spots of other VENERA landers. A "stalk" is the most interesting among them.

\section{REFERENCES}

[1] Arnold Selivanov, Yurii Gektin, Michail Gerasimov et al., Kosmicheskiye Issledovaniya 21 (1983) 176-182

[2] Leonid Ksanfomality, Doklady Physics 57 (2012) 367-372

[3] Leonid Ksanfomality, Solar System Research 46 (2012) 364-373

[4] Leonid Ksanfomality, Doklady Physics 57 (2012) 497-501

[5] Leonid Ksanfomality, Icarus 200 (2009) 367-373

[6] Alexander Kemurdzhian, Pavel Brodskii, Valeryi Gromov et al., Kosmicheskiye Issledovaniya 21 (1983) 323-330

[7] Charles Cockell, Planetary and Space Science 47 (1999) 1487-1501

[8] Barrie Jones "Life in the Solar System and Beyond" Springer (2004) Chichester

[9] Leonid Ksanfomality, Proceedings of the SPIE 4939 (2003) 169-173

[10] Leonid Ksanfomality, "Planeta Venera" Fizmatlit (1985) Moscow 